\title{
OPTIMIZATION USING TOPOLOGICAL DERIVATIVE AND BOUNDARY ELEMENT METHOD WITH FAST MULTIPOLE
}

\author{
L. M. Braga ${ }^{1}$, C. T. M Anflor ${ }^{2}$, E. L. Albuquerque ${ }^{1}$ \\ ${ }^{1}$ Departamento de Engenharia Mecânica, Universidade de Brasília, Campus Universitário \\ Darcy Ribeiro, Brasilia, DF, Brasil, (ㄹer@unb.br) \\ ${ }^{2}$ Engenharia Automotiva, Universidade de Brasília, Campus Gama, DF, Brasil, \\ (anflor@unb.br )
}

\begin{abstract}
The objective of this work is to compare topologies resulting from direct BEM (Boundary Element Method) with a BEM accelerated by Fast Multipole Method (FMM). A formulation of fast multipole boundary element (FMBEM) is introduced in order to turn the optimization process more attractive in the point of view of the computational cost. The formulation of the fast multipole is briefly summarized. A topological-shape sensitivity approach is used to select the points showing the lowest sensitivities, where material is removed by opening a cavity. As the iterative process evolves, the original domain has holes progressively removed, until a given stop criteria is achieved. A benchmark is investigated by imposing different FMBEM parameters. For effect of comparison the topology resulting from an analytical BEM optimization process is used. The topologies resulting due to this set of parameters imposed are presented. The CPU time $x$ DOF's are also investigated. The accelerated BEM demonstrated good feasibility in an optimization routine.
\end{abstract}

Keywords: Topology optimization, topological derivative, fast multipole method, boundary element methods.

\section{INTRODUCTION}

Although the Boundary Element Method (BEM) provides some facilities when modeling many problems its efficiency is not suitable for large-scale models. The BEM in general produces dense and non-symmetric matrices that, in spite of smaller in sizes, requires $\mathrm{O}(\mathrm{N} 2)$ operations to compute the coefficients. In order to solve the resulting system using direct solvers another $\mathrm{O}(\mathrm{N} 3)$ operations is also required. In order to overcome this inefficiency a coupling between Fast Multipole Method (FMM) and BEM is purposed. This will allow solving problems with several millions of degree of freedom (DOF's). Generally for large scales models the Finite Element Method (FEM) was indicated to solve models with several millions of DOF's, on the other side, the BEM has been limited to solving problems with a few thousands DOF's for many years. In the last years great efforts has been done by 
scientists to improve the BEM maintaining its all attractive, such as, easy mesh in modeling, small matrices of coefficients, no mesh dependency. The next step relies on expand the method to solve problems of large-scale. An example of large-scales is the topology optimization problem. As it is an iterative problem, a number of elements are always increasing because the material is being removed and a significantly number of DOF's must be solved. In the point of view of computational cost it should be a serious problem especially when the case under investigated has its statement in 3D optimization. During the last years many efforts have been done in order to accelerate the BEM for large-scales problems. As pioneers $[1,2]$ presented the FMM which promised the accelerating the solutions of BIE. The main goal was to reduce the CPU time in FMM accelerated BEM to $\mathrm{O}(\mathrm{N})$. Thereafter this new technique was applied for solving elasticity [3] and fluids [4] problems in large-scale. Some years after, [5] announced the FMM as one of the top algorithms in scientific computing that were developed in the 20th century. In this publication the authors had developed a complete tutorial which presents the basic concept and the main procedures in the FMM for solving boundary integral equations for 2D potential problems. The author [6] extended the FMM formulation for large-scale analysis of two-dimensional (2D) Stokes flow problems. For solving the dual Boundary Integral Equation (BIE) formulation, the author had employed a linear combination for velocity and the hipersingular BIE for traction to attain a better conditioning for the BEM system of equations. Some examples were presented and showed a good accuracy and efficiency of the proposed approach. Also [7] published the book entitle Fast Multipole Boundary Element Method where many instructions are given in order to provide fundamentals for others researches can implement this method. The FMM was implemented by [8] for solving the effective thermal conductivity (ETC) of random micro-heterogeneous materials using representative elements and FMBEM. The main goal of this paper is to implement the FMM in a topology optimization code. The idea relies on compare the performance of both methodologies, i.e., optimization with Direct BEM against FMBEM in the point of view of CPU time and resulting topologies. This paper is organized as follow: In Section 2 the main idea of TD is discussed and the analytical expressions for TD in Poisson problems are presented. In Section 3 and 4 the methods BEM and FMBEM for 2D potential problem is shown, respectively. In Section 5 some numerical examples and their respectively results are presented. Finally, in Section 6 this work is concluded and some discussions are carrying on.

\section{TOPOLOGICAL DERIVATIVE}

A topological derivative for Poisson Equation is applied in this work for determining the domain sensitivity. A simple example of applicability consists in a case where a small hole of radius $(\varepsilon)$ is open inside the domain. The concept of topological derivative consists in determining the sensitivity of a given function cost $(\psi)$ when this small hole is increased or decreased. The local value of TD at a point $(\overline{\bar{x}})$ inside the domain for this case is evaluated by:

$$
\stackrel{*}{D_{T}(\bar{x})}=\lim _{\varepsilon \rightarrow 0} \frac{\psi\left(\Omega_{\varepsilon}\right)-\psi(\Omega)}{f(\varepsilon)},
$$


where $\psi(\Omega)$ and $\psi(\varepsilon)$ are the cost function evaluated for the original and the perturbed domain, respectively, and $\mathrm{f}$ is a problem dependent regularizing function. By eq (1) it is not possible to establish an isomorphism between domains with different topologies. This equation was modified introducing a mathematical idea that the creation of a hole can be accomplished by single perturbing an existing one whose radius tends to zero. This allows the restatement of the problem in such a way that it is possible to establish a mapping between each other [9].

$$
\stackrel{*}{D_{T}(\bar{x})}=\lim _{\varepsilon \rightarrow 0} \frac{\psi\left(\Omega_{\varepsilon+\delta \varepsilon}\right)-\psi\left(\Omega_{\varepsilon}\right)}{f\left(\Omega_{\varepsilon+\delta \varepsilon}\right)-f\left(\Omega_{\varepsilon}\right)}
$$

where $\delta \varepsilon$ is a small perturbation on the holes's radius. In the case of linear heat transfer, the direct problem is stated as:

$$
\text { Solve } \quad\left\{u_{\varepsilon} \mid-k \Delta u_{\varepsilon}=b\right\} \text { on } \quad \Omega_{\varepsilon}
$$

subjected to:

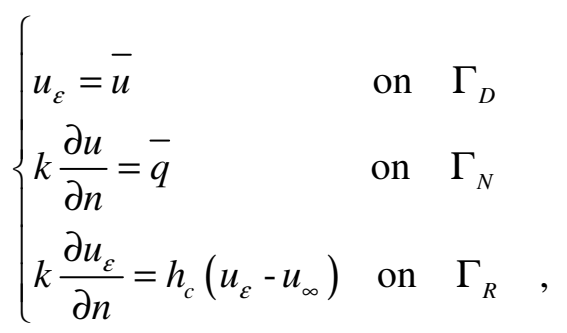

Where,

$$
h(\alpha, \beta, \gamma)=\underbrace{\alpha\left(u_{\varepsilon}-\bar{u}^{\varepsilon}\right)}_{\text {Dirichlet }}+\underbrace{\beta\left(k \frac{\partial u_{\varepsilon}}{\partial n}+\bar{q}^{\varepsilon}\right)}_{\text {Neumann }}+\underbrace{\gamma\left(k \frac{\partial u_{\varepsilon}}{\partial n}+h_{c}^{\varepsilon}\left(u_{\varepsilon}-u_{\infty}^{\varepsilon}\right)\right)}_{\text {Robin }}=0
$$

is a function which takes into account the type of boundary condition on the holes to be created $\left(u_{\varepsilon}, \frac{\partial u_{\varepsilon}}{\partial n}=q_{\varepsilon}\right.$ are the temperature and flux on the hole boundary, while $u_{\infty}^{\varepsilon}$ and $h_{c}^{\varepsilon}$ are the hole's internal convection parameters, respectively). After an intensive analytical work, it was developed explicit expressions for TD for problems governed by eq (3). Table 1 summarizes the final expressions for topological derivative, considering the three classical cases of boundary conditions on the holes.

Table 1. Topological derivative for the various boundary conditions prescribed on the holes.

\begin{tabular}{l|ll}
\hline \multicolumn{1}{c|}{ BOUNDARY CONDITION ON THE HOLE } & TOPOLOGICAL DERIVATIVE & EVALUATED AT \\
\hline $\begin{array}{l}\text { Neumann homogeneous boundary condition } \\
(\alpha=0, \beta=1, \gamma=0)\end{array}$ & $D_{T}(\overline{\bar{x}})=k \nabla u \nabla u-b u$ & $\bar{x} \in \Omega \cup \Gamma$ \\
$\begin{array}{l}\text { Neumann non-homogeneous boundary condition } \\
(\alpha=0, \beta=1, \gamma=0)\end{array}$ & $D_{T}(\bar{x})=-q_{\varepsilon} u$ & $\bar{x} \in \Omega \cup \Gamma$ \\
$\begin{array}{l}\text { Robin boundary condition } \\
(\alpha=0, \beta=0, \gamma=1)\end{array}$ & $D_{T}(\bar{x})=h_{c}^{\varepsilon}\left(u_{\varepsilon}-u_{\infty}\right)$ & $\bar{x} \in \Omega \cup \Gamma$ \\
$\begin{array}{l}\text { Dirichlet boundary condition } \\
(\alpha=1, \beta=0, \gamma=0)\end{array}$ & $D_{T}(\bar{x})=-\frac{1}{2} k\left(u-\bar{u}_{\varepsilon}\right)$ & $\bar{x} \in \Omega$ \\
$\begin{array}{l}\text { Dirichlet boundary condition } \\
(\alpha=1, \beta=0, \gamma=0)\end{array}$ & $D_{T}(\bar{x})=k \nabla u \nabla u-b \bar{u}_{\varepsilon}$ & $=$ \\
\hline
\end{tabular}




\section{BEM}

A brief review on Boundary element method using constant elements is summarized in this work. An initial domain is established with boundary conditions prescribed on its boundary. Considering a Laplace equation governing a 2D potential problems:

$$
\nabla^{2} u(x)=0, \forall x \in \Omega
$$

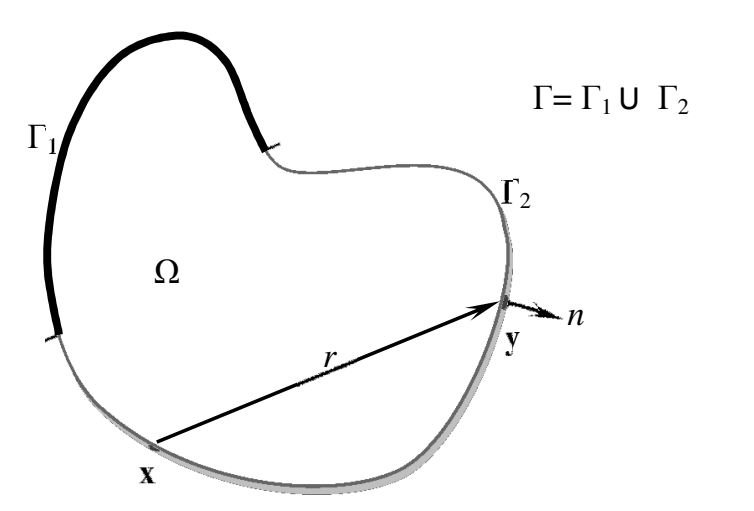

Fig.1 Domain $\Omega$ and its boundary $\Gamma$.

For a potential problem three kinds of boundary conditions may be imposed, Dirichlet, Neumann and/or Robin. For this presentation the first and second boundary conditions are imposed,

$$
\begin{aligned}
& u(x)=\bar{u}(x), \forall x \in \Gamma_{1} ; \\
& q(x)=\frac{\partial u}{\partial \eta}(x)=\bar{q}(x), \forall x \in \Gamma_{2}
\end{aligned}
$$

Where $\mathrm{u}$ is the potential field in domain $(\Omega), \Gamma$ is the boundary of $\Omega, \mathrm{n}$ is the outward normal. Note that the barred quantities are the values imposed by the boundary conditions on the boundary. The solution of eq.(6) under boundary conditions as eq.(7) is:

$$
u(x)=\int_{S}\left[u^{*}(x, y) q(y)-q^{*}(x, y) u(y) d S(y), \forall x \in \Omega\right.
$$

Where $u^{*}(x, y)$ and $q^{*}(x, y)$ are the Green's function for 2D problems

$$
q^{*}(x, y)=\frac{\partial u^{*}(x, y)}{\partial \eta(y)}=\frac{1}{2 \pi r} \frac{\partial r}{\partial \eta}
$$

and $r$ represents the distance between the collocation point $\mathbf{x}$ and the field point $\mathbf{y}$, as depicted in fig.1. Taking $\mathbf{x}$ belongs to the $\Gamma$ the classic BIE formulation of BEM [10] is obtained as:

$$
C(x) u(x)=\int_{S}\left[u^{*}(x, y) q(y)-q^{*}(x, y) u(y) d S(y), \forall x \in \Gamma ;\right.
$$

If the boundary is smooth in the collocation point $\mathbf{x}$, the coefficient $\mathrm{C}(\mathbf{x})=1 / 2$. The next step consists in discretize the boundary $\Gamma$ using $\mathrm{N}$ constant elements as illustrated by fig. 2 . 


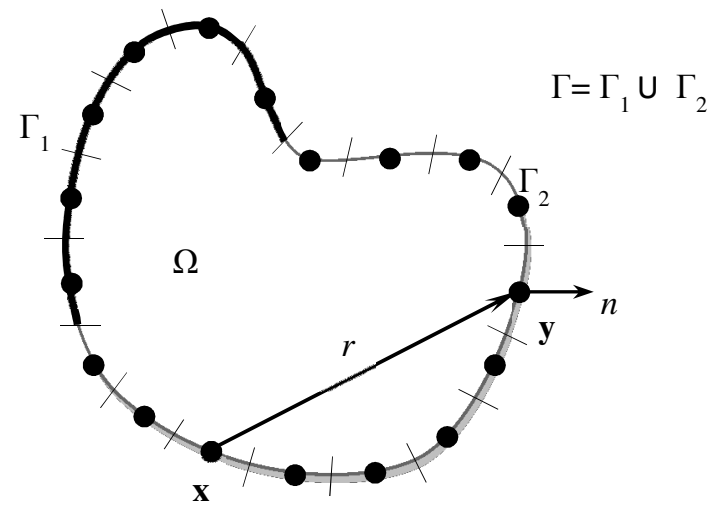

Figure 2. Discretization of the boundary $\Gamma$ using constant elements.

The discretized equation of BIE is now presented as,

$$
\frac{1}{2} u_{i}=\sum_{j=1}^{N}\left[G_{i j} q_{j}-\sum_{j=1} \hat{H}_{i j} u_{j}\right], i=1,2,3, \ldots, N ;
$$

Where the $u_{j}$ and $q_{j}(\mathrm{j}=1,2, \ldots, \mathrm{N})$ are the nodal values of the $\mathrm{u}$ and $\mathrm{q}$ in the element $\Delta \mathrm{S}_{\mathrm{j}}$, respectively. Applying the boundary conditions (2) at each node and switching the columns for grouping the unknowns variables one finds,

$$
A \lambda=B
$$

And where $\mathbf{A}$ is the coefficients matrix, $\lambda$ the unknown vector and $\mathbf{b}$ the known righthand side vector.

\section{FAST MULTIPOLE BOUNDARY ELEMENT}

BEM uses the Green's functions as the weighting function on its formulation which increase the accuracy when compared with another numerical techniques [10]. As a result the spatial dimension is reduced by one. Additionally, the computational cost of a traditional BEM direct can be reduced by using the FMBEM. The goal of FMM relies on translating node-to-node interactions to cell-to-cell interactions. These cells have a hierarchical structure called as tree while the small ones are called as leaves. FMM employs iterative equations solvers (GMRES) where matrix-vector multiplications are calculated using fast multipole expansions. As iterative equations are used some parameters for the FMM, such as, maximum number of elements allowed in a leaf (maxl) and in the tree structure (levmax), number of terms in multipole expansion (nexp) and local expansion (ntylr), and also the GMRES solution convergence (tol) must be set. The expansions used for 2D potential problem for FMM are briefly summarized as table 2. Further details about the analytical derivations should be attained in [5,7]. 
Table 2. Resume of the M2M, M2L and L2L expansions.

\begin{tabular}{l|c}
\hline & Kernel $u^{*}$ \\
\hline M2M & $M_{k}\left(z_{c}^{\prime}\right)=\sum_{l=0}^{k} I_{k}\left(z-z_{c}^{\prime}\right) M_{l}\left(z_{c}\right)$ \\
M2L & $\int_{s_{0}} u^{*}\left(z_{0}, z_{c}\right) q(z) d \Gamma(z)=\sum_{l=0}^{\infty} L_{l}\left(z_{L}\right) I_{l}\left(z_{0}-z_{L}\right)$ \\
L2L & $\int_{s_{0}} u^{*}\left(z_{0}, z\right) q(z) d \Gamma(z)=\sum_{l=0}^{\infty} L_{l}\left(z_{L^{\prime}}\right) I_{l}\left(z_{0}-z_{L^{\prime}}\right)$ \\
\hline
\end{tabular}

The main idea of the fast multipole BEM should be briefly described as:

- Step 1 - Discretization. The domain $\Omega$ is discretized as BEM approach conventional way.

- Step 2 - Determine a tree structure of the boundary element mesh. A square covering the discretized domain $\Gamma$ is considered. This square is classified as a cell of level 0 . This parent cell is divided into four child cells, now classified as level 1 . This procedure is iteratively done until a stop criteria is achieved. This criteria is achieved when the number of elements imposed by the user in that cell is reached. A cell having no child cells is call as leaf, which are in grey as fig.1.

- Step 3 - Compute the moments on all cells. This step is also known as upward pass. The moments are computed on all cells. If a leaf is under consideration the moments is calculated directly by using $M_{k}\left(z_{c}\right)=\int_{S_{c}} I_{k}\left(z-z_{c}\right) q(z) d S(z)$, where $\mathrm{S}_{\mathrm{c}}$ is the set of elements contained in the leaf and $\mathrm{z}_{\mathrm{c}}$ the centroid of the leaf. For the parent cell the M2M translation is applied and the moments are summed on its four child cells. The M2M equation is presented by tab. 2 and $z_{c}$ is the centroid of the parent cell while the $z_{c}$ represents the centroid of a child cell.

- Step 4 - Specify cells with interactions (M2L), far cells (L2L), adjacent cells and cell C. This step was named as Downward pass. In this step a classification is done in order to define the distribution of all cell around a defined Cell C. Adjacent cells are those that level $l$ have at least one common vertex. Cells well separated at a level $l$ are those who are not adjacent at level $l$ but their parents cells are adjacent at level $l$-1.The interaction list of $\mathrm{C}$ is a list of all well-separated cells from a level $l$ cell $\mathrm{C}$. Far cells of $\mathrm{C}$ are those that their parent cells are not adjacent to the parent cell of $\mathrm{C}$. The local expansion associated with a cell $\mathrm{C}$ is calculated by the use of the M2L translation. The L2L translation is calculated for the parent cell of $\mathrm{C}$ with the expansion point being shifted from the centroid of C's parent cell to that of C. Considering the cell $\mathrm{C}$ at level 2, the M2L translation is used to compute the coefficients of the local expansion.

- Step 5 - Evaluation of the integrals.

- Step 6 -Iterations of the solutions. The unknown solution vector $\lambda$ in the system $A \lambda=B$ is update by the iterative solver and continues for all levels to evaluate the subsequent matrix and vector multiplication $A \lambda$ until the solution $\lambda$ converges to a defined tolerance. 
Figure 1 depicts the basic idea of the FMM in steps.

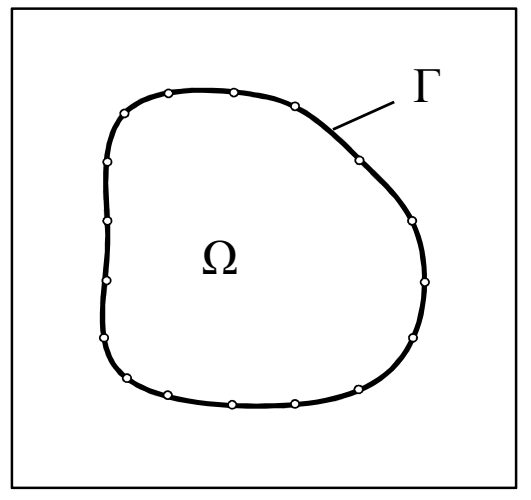

Step 1 - Initial Domain - level 0

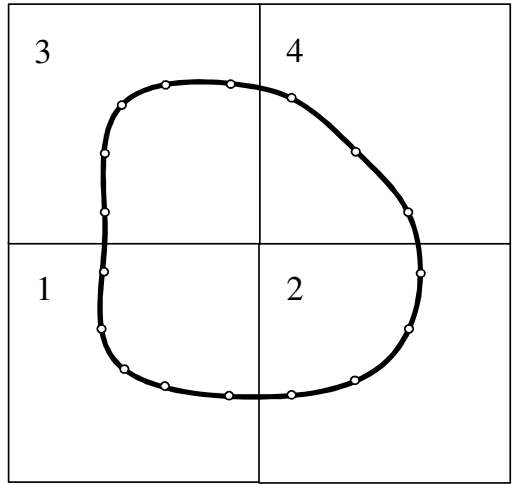

Step 2 Discretization - Level 1

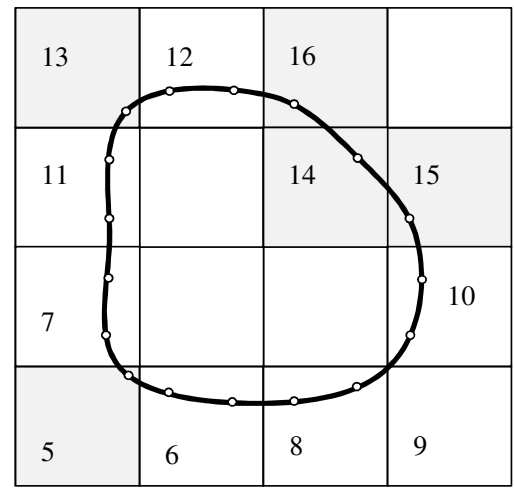

Step 2 Discretization - Level 2

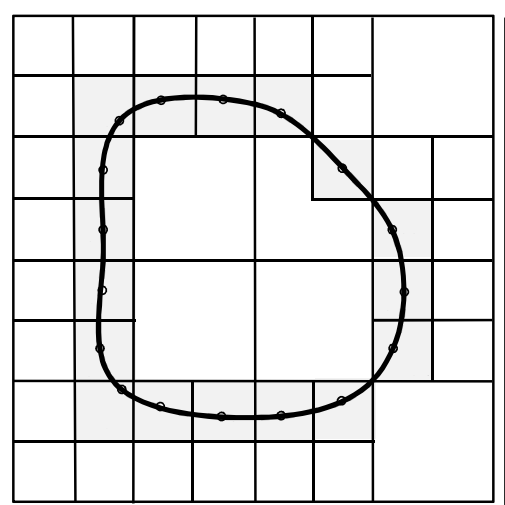

Step 2 - Discretization - Level 3

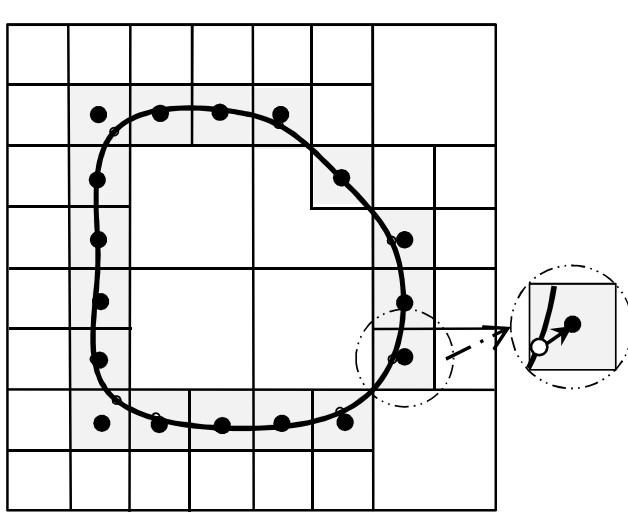

Step 3 - Multipole expansion

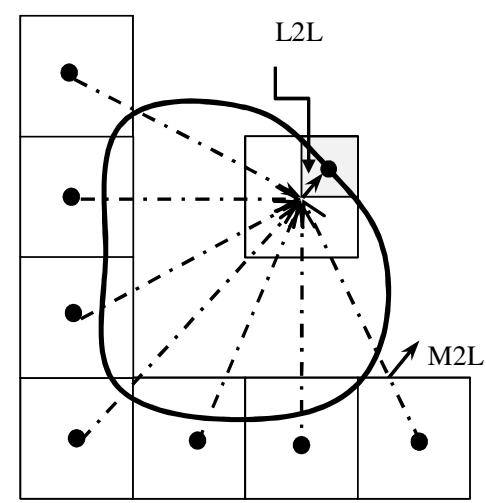

Step $4-$ M2L and L2L - level 2

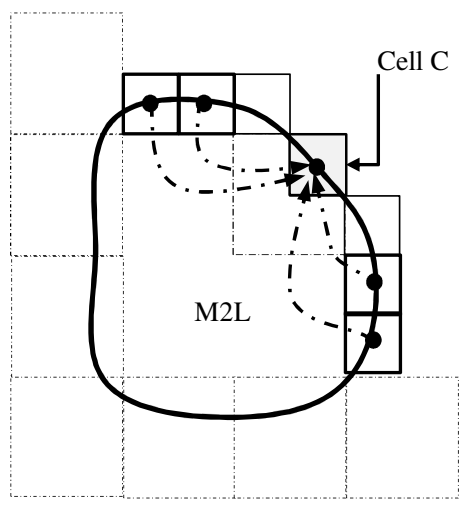

Step 4 - M2L level 3
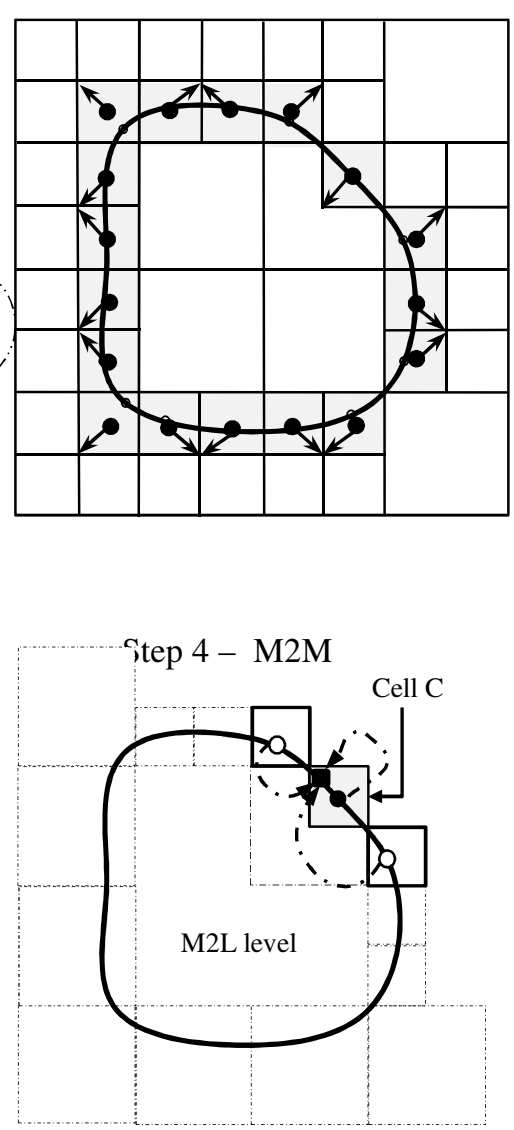

Step 5 - Evaluation of all integrals

Figure 1. FMM Scheme.

\section{NUMERICAL RESULTS}

The high computational effort involved in an optimization process motivates the implementation of FMM in order to maintain those attractive characteristics when coupling BEM and DT [11]. The topology optimization process is carried out using an Intel Pentium 
core 2 Duo with $4 \mathrm{~GB}$ of RAM and 2,93GHz. This section presents one example that demonstrates the application of the proposed method. The results obtained for each case are compared with Direct BEM versus FMBEM. During the optimization process the computational cost, number of DOFs and volume were taken into account. For a specific iteration the respective intermediary topology is illustrated. The iterative process was halted when a given amount of material was removed from the original domain. In all cases the total potential energy was used as the cost function. A regularly-spaced grid of internal points was generated automatically, taking into account the radius of the holes created during each iteration. The radius was obtained as a fraction of a reference dimension of the domain $(r=\omega$ $1_{\text {ref }}$ ). In all cases $1_{\text {ref }}=\min$ (height,width) was adopted. The objective in all cases was to minimize the material volume. The current volume of the domain $\left(\mathrm{V}_{\mathrm{f}}\right)$ was checked at the end of each iteration until a reference value was achieved $\left(V_{f}=\varphi V_{0}\right.$, where $V_{0}$ represents the initial volume and $\varphi$ a defined percentage of material to be removed).

\subsection{Heat Conductor}

This example refers to a square domain subjected to low temperature boundary conditions on its corners and a decentralized high temperature b.c. at the left surface. The problem is illustrated as Fig.2, where $\mathrm{T}_{\mathrm{H}}$ is the high temperature $(373 \mathrm{~K})$ and $\mathrm{T}_{\mathrm{L}}$ is the low temperature $(273 \mathrm{~K})$.

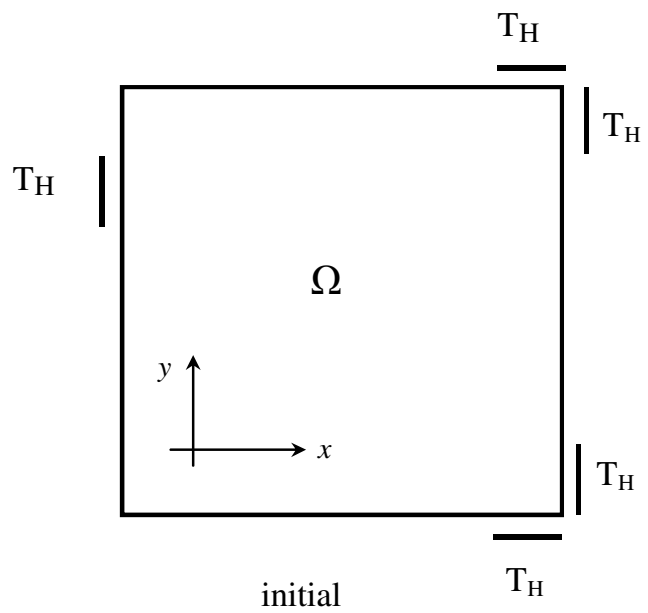

Figure 2. Heat conductor boundary conditions.

The remaining boundaries and the holes opened during the optimization process are insulated. The stop criteria was set until $\mathrm{V}_{\mathrm{f}}=0.6 \mathrm{~V}_{0}$ is achieved. In order to evaluate the different resulting topologies due to the FMMBEM parameters three cases are under investigation. All cases are always compared with the topology resulted by using direct BEM namely by case (f). For the case (f) it was used constant elements and all integrations were 
performed analytically. Figure 3 depicts these topologies comparatives and also presents the parameters used to set the FMBEM for each case. As presented in fig. 3 it is possible to see the refined mesh grid of internal points. Instead FMBEM being evaluated only on the boundary, the internal points are recovered as the traditional BEM direct. Fortunately, the presented code has implemented the facility of generating internal points only near the boundaries (offset) or in all domain. Obviously, when dealing with domains with a significantly internal sensitivity an evaluation on all domain is required. A good recommendation is to use both numerical artifices, i.e. some iterations with an offset of internal points and a predefined intermediary iteration which takes in to account a complete grid over the domain, see fig. 3. It is also important to notice that this procedure is not possible to be done in the finite element method due to its features of mesh domain. This kind of numerical implementation also contributes to the characteristics provided from BEM.
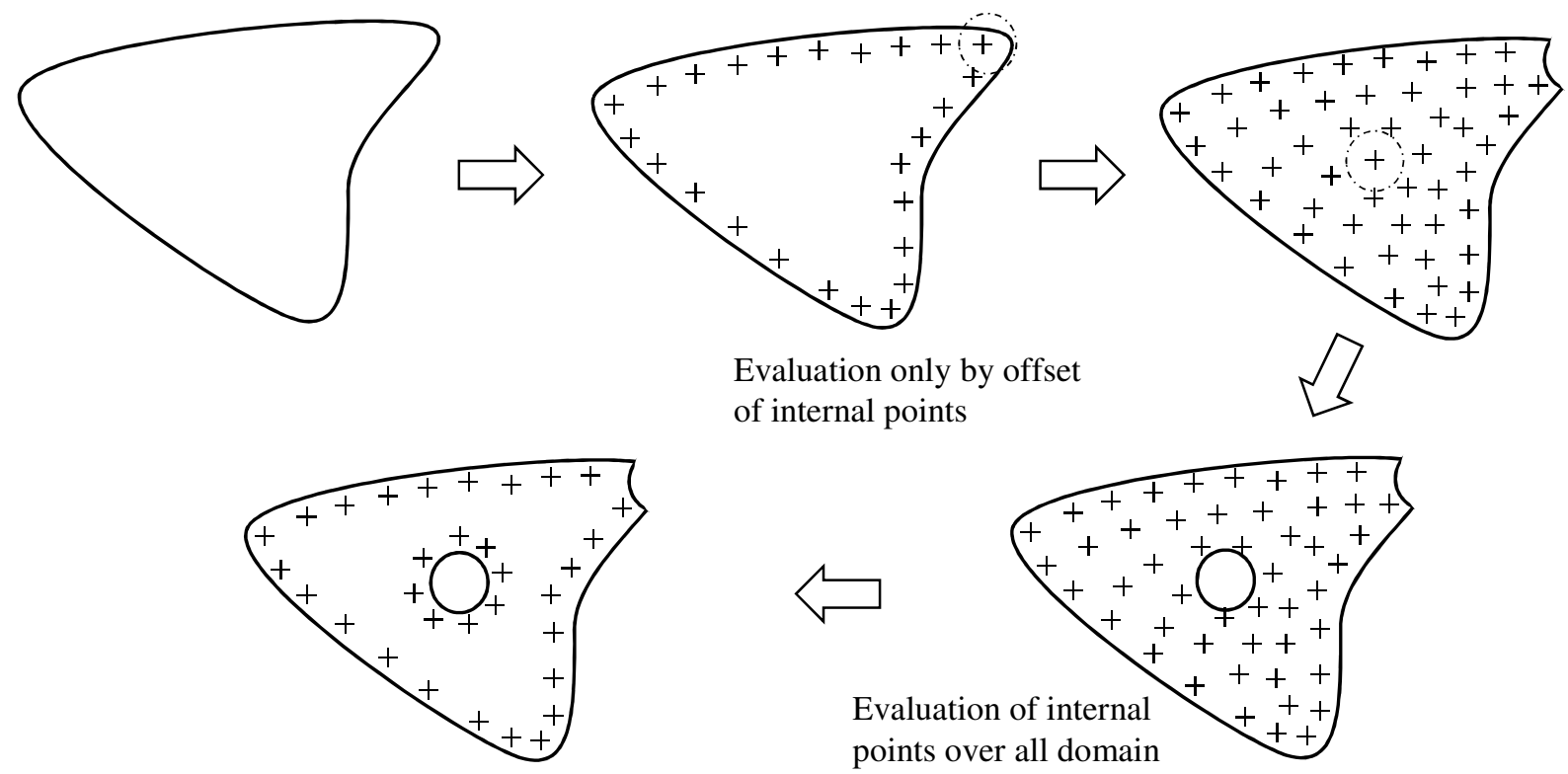

Evaluation only by offset of internal points

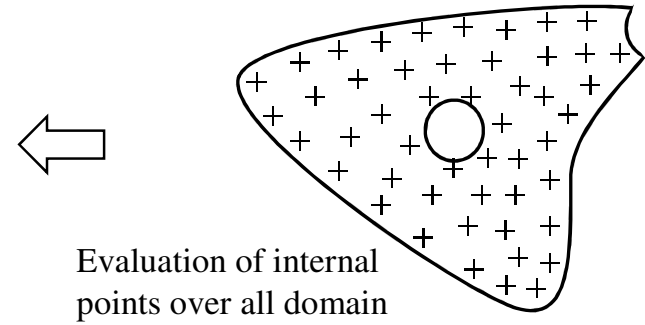

Figure 3. Internal Points: Evaluation by offset and over all domain.

The first four topologies showed a slightly difference when compared with case (f), due the parameters of FMBEM employed, see fig.4. From now on, only with case (c) and case (f) will be refereed. Case (c) resulted a topology that match perfectly with those resulted by using Direct BEM and it is possible to compare temperature of both by fig. 5. Also it is important to note that both topologies attained the same volume at the same iteration.

The CPU time x DOF for case (e) and case (f) are presented as fig.6. During the optimization process a number maximum of approximately 3500 elements were evaluated. It is possible to verify that the performance of FMBEM is superior when an elevate number of elements are being integrated. An interesting evaluation relies on determining the intersection between the curves. 


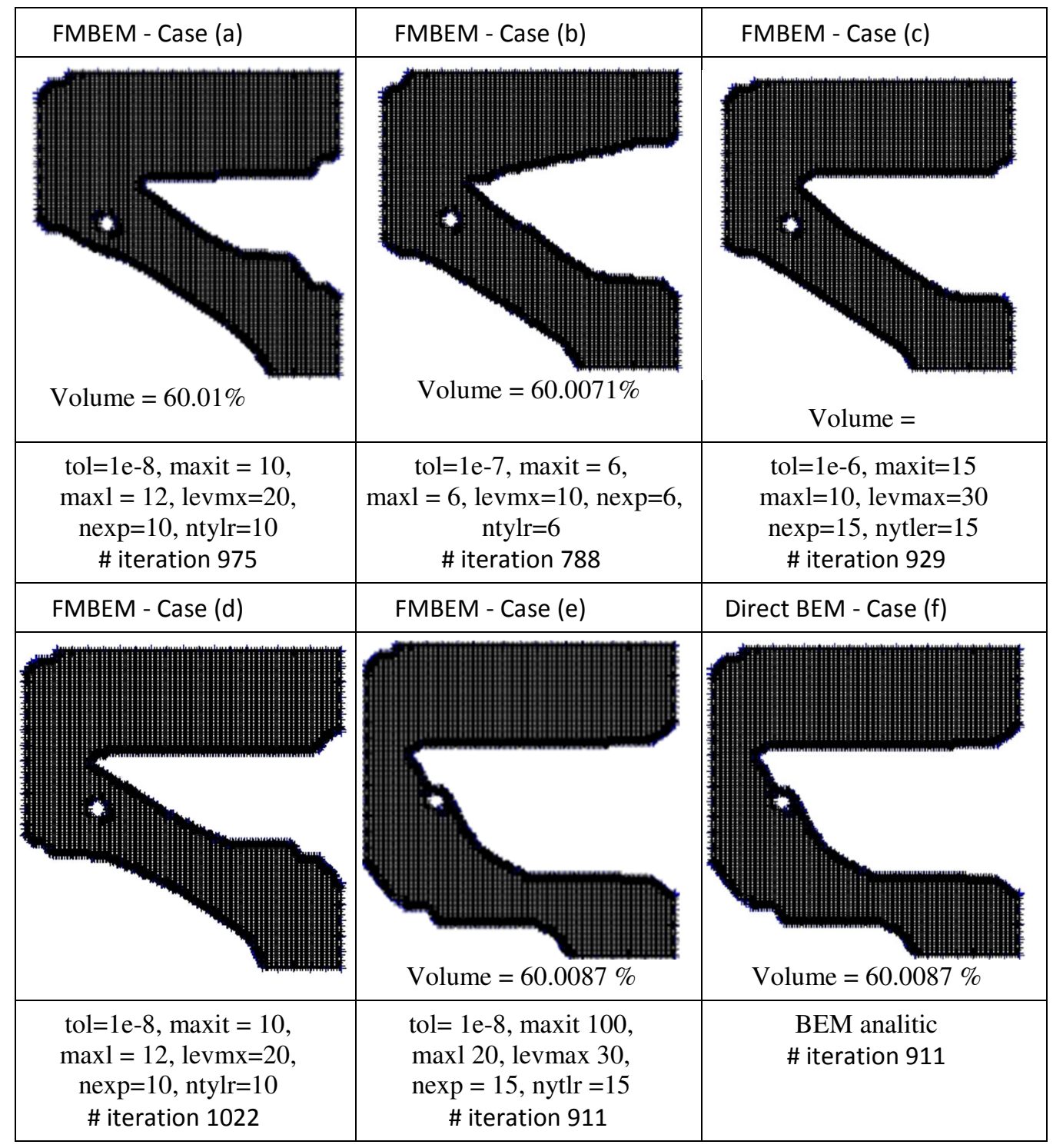

Figure 4 . Comparative between topologies with approximately $60 \%$ of volume.

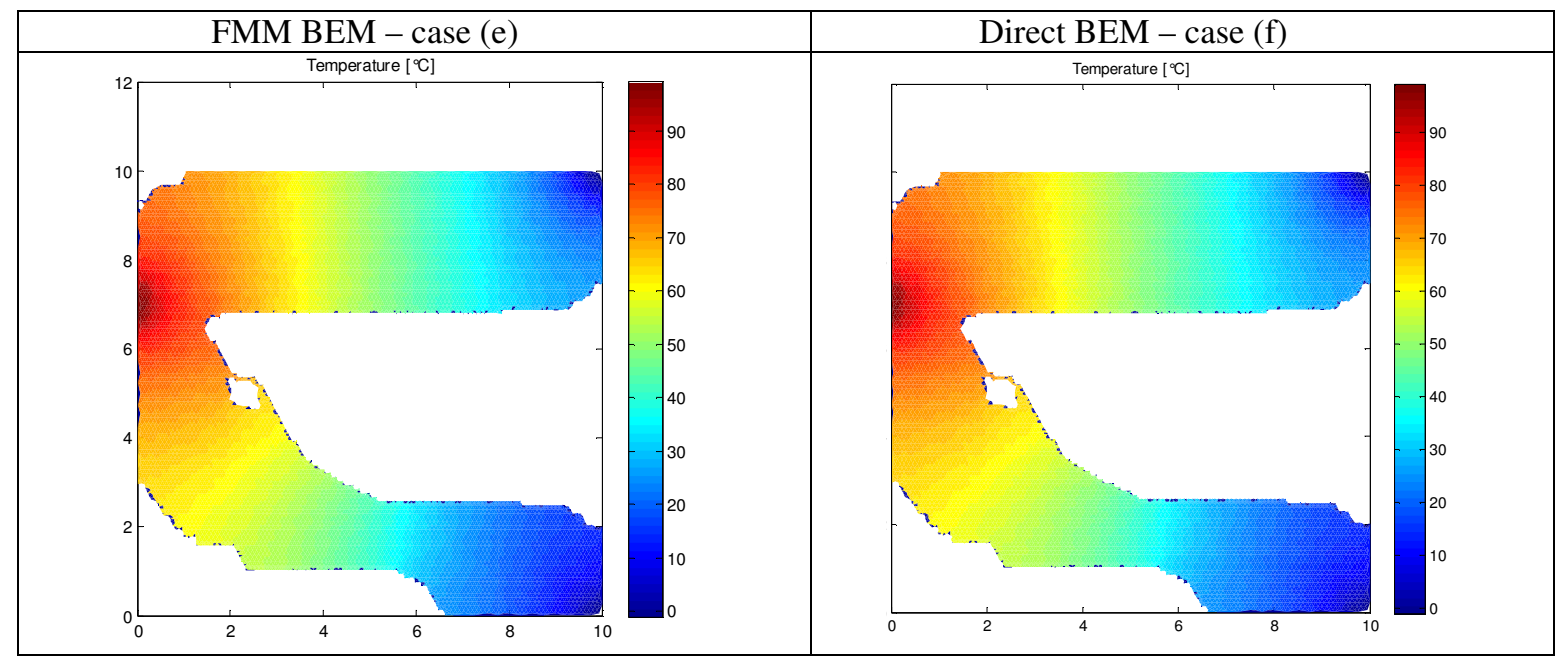

Figure 5. Color map for case (e) and case (f). 


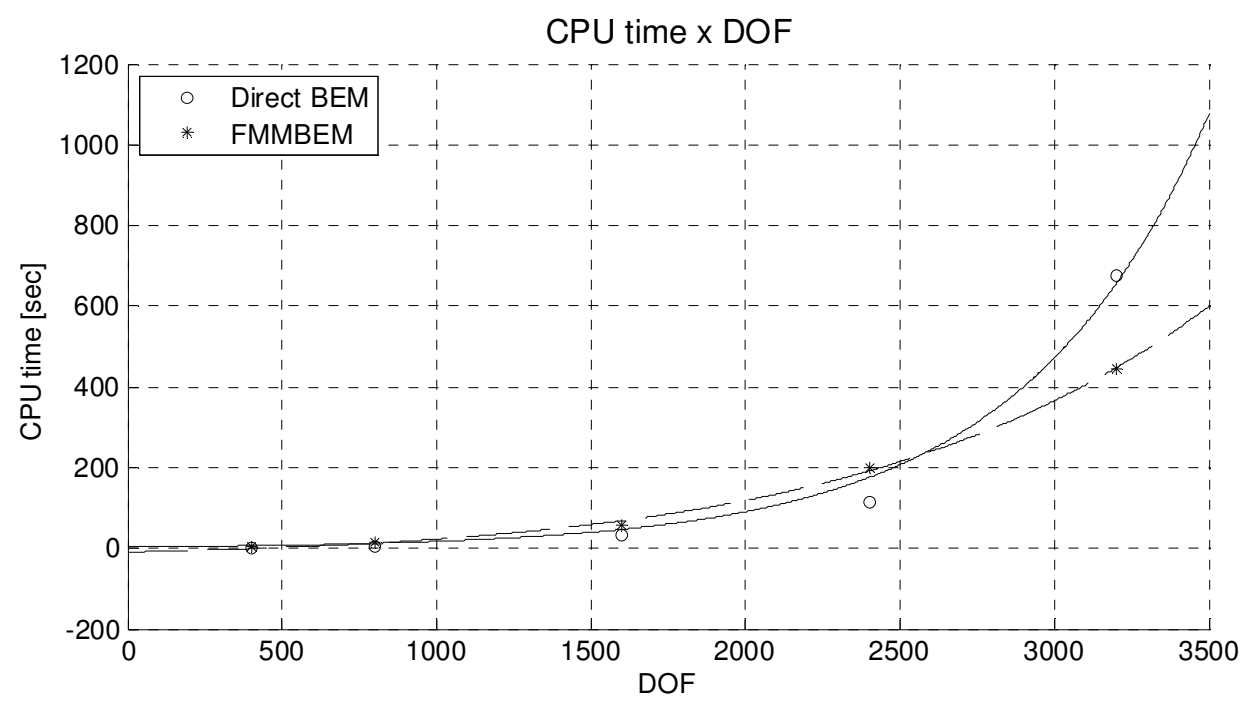

Figure 6. A comparative: CPU time $x$ DOF.

\section{CONCLUSIONS}

In the numerical analysis the computational cost is an important issue. Regardless of many attractive the boundary element method is not widely released in the commercial codes because the traditional method is not recommended for solving large-scale problems. This argument is increased when considering the iterative optimization process where the problem must be evaluated several times. In order to overcome this issue the FMBEM was implemented in the topological optimization code. The resulting topology of a benchmark using FMBEM was compared with the final topology obtained by using direct BEM. The CPU times for both cases were compared until the final topologies have been reached. The final topologies for case (e) and (f) have shown good agreement once the FMBEM parameters were adjusted. The results suggest that BEM is more efficient for problem with a low discretization, i.e., low DOF's. As the iterative process evolves the number of elements increases significantly and the FMBEM takes advantage over the BEM direct. Some remarks must be taken in account, such as; while the iterative process doesn't reach about 3500 DOF's the BEM directly is recommended. When this number of DOF's is exceeded a flag (previously implemented in the code) must be turned on and the process be carry on using FMBEM. Another interesting remark relies on the fact that the final shape of the resulting topology depends significantly on the parameters set for the FMBEM. Finally, with FMBEM topologies very well refined must be attained with a simple CPU without needing parallel computation.

\section{REFERENCES}

[1] Roklin V., "Rapid Solution of integral equations of classical potential theory". J. Comput Phys, 60, 187-207, 1985. 
[2] Greengard L.F., "The rapid evaluation of potentials fields in particle systems", Cambridge: The MIT Press, 1988.

[3] Peirce A.P., Napier J.A.L. A spectral multipole method for efficient solutions of large scale boundary element models in elastostatics. Int J Numer Meth Eng. 38, 4009-4034, 1995.

[4] Mammoli A. and Ingber M., "Stokes flow around cylinders in a bounded two-dimensional domain using multipole accelerated boundary element methods", International Journal for Numerical Methods in Engineering, 44, 897-917 (1999).

[5] Liu Y.J., Nishimura N., "The fast multipole boundary element method for potential problems: A tutorial”. Engineering Analysis with Boundary Elements, 30, 371-381, 2006.

[6] Liu Y.J., "A new fast multipole boundary element method for solving 2-D Stokes flow problems based on a dual BIE formulation". Engineering Analysis with Boundary Elements, 32, 139-151, 2008.

[7] Liu Y.J., "Fast Multipole Boundary Element Method: Theory and applications in Engineering", Cambridge 2009.

[8] Dondero M., Cisilino A.P., Carella J.M., Tomba J. P., "Effective thermal conductivity of functionally graded random micro-heteregeneous materials using representative volume element and BEM". International Journal of Heat and Mass transfer, 54, 3874-3881 2011.

[9] Feijóo R., Novotny A., Taroco E., Padra C., “The topological derivative for the Poisson's problem". Mathematical Model and Methods in Applied Sciences, 13, 1825-1844, 2003.

[10] Wrobel L.C. and Aliabadi M.H, "The Boundary Element Method, Vol2: Applications in Solids and Structures", Wiley 2002.

[11] Anflor C.T.M., Marczak R.J., "Topological optimization of anisotropic heat conducting devices using Bézier-smoothed Boundary Representation”. Computer Modeling in Engineering \& Sciences (Print), 1970, 151-168, 2011. 